\title{
Low-dose-rate brachytherapy for patients with transurethral resection before implantation in prostate cancer. Long- term results
}

\author{
Pedro J. Prada ${ }^{1}$, Javier Anchuelo ${ }^{1}$, Ana García Blanco ${ }^{1}$, Gema Payá ${ }^{1}$, Juan Cardenal ${ }^{1}$, Enrique Acuña \\ 1, María Ferri ${ }^{1}$, Andrés Vázquez ${ }^{2}$, Maite Pacheco, Jesica Sanchez ${ }^{2}$ \\ ${ }^{1}$ Department of Radiation Oncology, Hospital Universitario Marqués de Valdecilla, Santander, Cantabria, \\ Spain; ${ }^{2}$ Department of Radiation Physics, Hospital Universitario Marqués de Valdecilla, Santander, \\ Cantabria, Spain
}

\section{ABSTRACT}

Objectives: We analyzed the long-term oncologic outcome for patients with prostate cancer and transurethral resection who were treated using low-dose-rate (LDR) prostate brachytherapy.

Methods and Materials: From January 2001 to December 2005, 57 consecutive patients were treated with clinically localized prostate cancer. No patients received external beam radiation. All of them underwent LDR prostate brachytherapy. Biochemical failure was defined according to the "Phoenix consensus". Patients were stratified as low and intermediate risk based on The Memorial Sloan Kettering group definition.

Results: The median follow-up time for these 57 patients was 104 months. The overall survival according to Kaplan-Meier estimates was $88 \%( \pm 6 \%)$ at 5 years and $77 \%$ $( \pm 6 \%)$ at 12 years. The 5 and 10 years for failure in tumour-free survival (TFS) was $96 \%$ and respectively $( \pm 2 \%)$, whereas for biochemical control was $94 \%$ and respectively $( \pm 3 \%)$ at 5 and 10 years, $98 \%( \pm 1 \%)$ of patients being free of local recurrence. A patient reported incontinence after treatment (1.7\%). The chronic genitourinary complains grade I were 7\% and grade II, 10\%. At six months 94\% of patients reported no change in bowel function.

Conclusions: The excellent long-term results and low morbidity presented, as well as the many advantages of prostate brachytherapy over other treatments, demonstrates that brachytherapy is an effective treatment for patients with transurethral resection and clinical organ-confined prostate cancer.

\section{ARTICLE INFO}

\section{Key words:}

Brachytherapy; Transurethral Resection of Prostate; Prostatic Neoplasms

Int Braz J Urol. 2016; 42: 47-52

Submitted for publication: August 25, 2014

Accepted after revision: March 19, 2015

\section{INTRODUCTION}

Brachytherapy has rapidly gained popularity as an accepted, effective and safe therapy for localized prostate cancer. There are robust follow-up data beyond 10 years that show similar biochemical control rates to radical prostatectomy and external beam radiotherapy (1-3).
Many patients with preexisting lower urinary tract symptoms have been considered poor candidates for seed implants; however there have been few rigorous studies of the contraindications for brachytherapy. Several authors $(4,5)$ reported a higher risk of post-implant urinary incontinence in patients with a prior transurethral prostate resection (TURP). 
Transurethral prostate resection, developed in 1930, is a surgical procedure which consists on removal of the prostate parenchyma proximal to the verumontanum and distal to the bladder neck as a treatment for urinary obstruction. It is done without penetrating the prostatic capsule. The incontinence rate from TURP alone is low, ranging from $1 \%$ to $5 \%(6)$.

There has been little research on the safety and effectiveness of low dose rate brachytherapy performed in patients with prior TURP. The objective of the present study was to report the clinical outcome, side-effects and complications after permanent implantation of 125 I seeds for early prostate cancer in patients with a prior TURP with up to 10 years of follow-up.

\section{MATERIALS AND METHODS}

\section{Selection of patients}

In all, 57 patients with a TURP prior to brachytherapy were treated between January 2001 and December 2005; the median (range) follow-up was 104 (11-154) months. Patients were staged according to the American Joint Committee on Cancer $6^{\text {th }}$ edition clinical staging guidelines (7) using a directed history, physical examination and TRUS. All patients had their serum PSA level measured and Gleason score histological grading. The tumor characteristics are shown in Table-1.

In all patients TURP was done some months before brachytherapy (mean 70 months, range 4-132 months). All patients underwent small or medium not large TURP. The mean prostate volume, as measured by ultrasound before brachytherapy was 36cc (range 12-66cc). The mean resected volume was small (15g). The resected volume was noted at the time of brachytherapy but did not cause any technical problem to the seed implant to get enough tissue $(>1 \mathrm{~cm})$ at TURP level.

\section{Definition groups}

The Memorial Sloan Kettering group definition (8) was used to classify patients into risk groups; low-risk patients were T1c or T2a, with a PSA level of $\leq 10 \mathrm{ng} / \mathrm{mL}$ and Gleason score $\leq 6$; intermediate risk was T2b, PSA level 11-20ng/mL or Gleason score $\leq 7$; and high risk was $\geq \mathrm{T} 2$ c, PSA
level>20ng/mL or Gleason score $>7$, or two intermediate-risk criteria.

\section{Hormonal Therapy}

In our patient population, 40\% received hormone therapy before brachytherapy; this treatment was prescribed by the urologist, waiting for the definitive brachytherapy treatment. Hormonal therapy was given for 3 months and then stopped. The mean prostatic volume at implantation was 36 (12-66 cc).

\section{Treatment}

All patients received brachytherapy alone with I-125. The prescription dose was $145 \mathrm{~Gy}$ to the reference isodose (100\%) according to the TG43 (9). The target volume of the implant was the prostate gland plus a $2-5 \mathrm{~mm}$ peri-prostatic area.

The technique used in the implantation was based on intra-operative planning with real-time dynamic dose calculation with peripheral loading. The implantation technique has been previously described $(10,11)$.

To decrease rectal toxicity, transperineal hyaluronic acid injection into the peri-rectal fat was used to consistently displace the rectal wall away from the radiation sources in 6 patients. We considered that the increase in distance (mean $2 \mathrm{~cm}$ along the length of the prostate) would be enough to provide a significant reduction in radiation dose from LDR brachytherapy (12).

Patients were followed with symptom assessment and PSA determinations every 3 months for the first year, every 6 months for the second year and yearly thereafter.

\section{Toxicity}

Morbidity was reported according to the Common Terminology Criteria for Adverse Events (CTCAE 4.0). Toxicity and sexual side effects was scored by the physician.

\section{Statistical considerations}

Distant metastases disease was defined by an imaging study or physical examination that demonstrated cancer outside of the prostate and its regional nodes. Failure in tumor-free survival (TFS) analyses was represented as detection of 
Table 1 - Patient and tumor characteristics ( $n=57)$.

\begin{tabular}{|c|c|}
\hline Characteristics & $\mathrm{N}^{0}$ Patients $(\%)$ \\
\hline \multicolumn{2}{|l|}{ Stage } \\
\hline$\leq \mathrm{T} 2 \mathrm{a}$ & $50(88 \%)$ \\
\hline $\mathrm{T} 2 \mathrm{~b}$ & $7(12 \%)$ \\
\hline \multicolumn{2}{|l|}{ Gleason score } \\
\hline$\leq 6$ & $48(84 \%)$ \\
\hline$=7$ & $8(14 \%)$ \\
\hline$>7$ & $1(2 \%)$ \\
\hline \multicolumn{2}{|c|}{ Pretreatment PSA (ng/mL) } \\
\hline$\leq 10$ & $41(72 \%)$ \\
\hline $10.1-20$ & $15(26 \%)$ \\
\hline$>20$ & $1(2 \%)$ \\
\hline \multicolumn{2}{|c|}{ Mean: 9/Median 8 (1.4-47) } \\
\hline \multicolumn{2}{|c|}{ Adjuvant hormonal ablation } \\
\hline Yes & $23(40 \%)$ \\
\hline No & $34(60 \%)$ \\
\hline \multicolumn{2}{|l|}{ Age at diagnosis (year) } \\
\hline$\leq 60$ & $12(12 \%)$ \\
\hline $61-70$ & $25(44 \%)$ \\
\hline$>70$ & $25(44 \%)$ \\
\hline \multicolumn{2}{|l|}{ Risk Level } \\
\hline Low Risk & $48(84 \%)$ \\
\hline Intermediate Risk & $8(14 \%)$ \\
\hline High Risk by PSA & $1(2 \%)$ \\
\hline \multicolumn{2}{|c|}{ Gland Vol. Implant (cc): Mean: 36/Median 35 (12-66) } \\
\hline
\end{tabular}

local and/or systemic tumor relapse. Biochemical failure was defined according to the "Phoenix definition" (13) consensus panel statement. Estimated likelihood of events was calculated by the Kaplan-Meier method from the time of completion of brachytherapy procedure. The statistical significance of the difference between estimated event-free curves was calculated with the Log Rank test. Multivariate analysis was performed using the Cox proportional hazards model (14).

\section{RESULTS}

For the entire cohort of 57 patients, 3 had evidence of biochemical relapse, 2 had a clinical relapse and 1 died from prostate cancer; 6 patients died of other illnesses.

The overall survival according to Kaplan-Meier estimates was $88 \%( \pm 6 \%)$ at 5 years and $77 \%( \pm 6 \%)$ at 12 years. The 5 and 10 years for failure in tumor-free survival (TFS) were 96\% and 96\% $( \pm 2 \%)$ respectively, whereas for biochemical control was $94 \%$ and $( \pm 3 \%)$ at 5 and 10 years respectively, $98 \%( \pm 1 \%)$ of patients being free of local recurrence (Figure-1).

Cox proportional-hazards regression revealed no statistical significant association for clinical T stage, Gleason score, pretreatment PSA, age, brachytherapy dose (D90), hormonal ablative treatment and biochemical failure.

The actuarial biochemical control with Gleason score was 95\% and 89\% for patients with Gleason score of $\leq 6$ and 7 , respectively $(\mathrm{P}=0.4)$. The correlation with pretreatment PSA the biochemical control was 97\% and 89\% for patients with PSA of $\leq 10$ and $>10 \mathrm{ng} / \mathrm{ml}$, respectively $(\mathrm{P}=0.26)$.

T stage was not significant $(\mathrm{P}=0.38)$ for biochemical control (100\% for $\leq \mathrm{T} 2 \mathrm{a}$ and $93 \%$ for $\mathrm{T} 2 \mathrm{~b}$ ).

Mean patient age was 69 years (range 5577). The actuarial analysis of biochemical control at ages less than 61, 61 to 70 and greater than 70 years demonstrated no significant difference, as younger and older patients benefited equally $(\mathrm{P}=0.26)$.

The actuarial biochemical control was the same 93\% ( $\mathrm{P}=0.37)$, in patients who received hormones and in those who did not.

Increasing the dose received by $90 \%$ of the prostate volume (D90) from $\leq 160$ Gy and $>160$ Gy was not associated with improved biochemical control $(\mathrm{P}=0.37)$.

All 57 patients were discharge from the center the same day of the procedure between 6-8 hours of implantation. All patients have been seen in follow-up and the CTCAE toxicity criteria were utilized to score acute and late complications.

\section{Acute and Chronic Urinary Toxicity}

Moderate increase in urinary irritation (urethritis) occurred in the third month after treatment; the acute GU grade II toxicity was $9 \%$.

The incontinence rate prior to brachytherapy grade I were 9\% (5 patients) and grade II, $3 \%$ (2 patients). Only a patient without prior 
Figure 1. Actuarial analysis of all 57 patients for overall survival, tumor-free survival and biochemical control and local control.

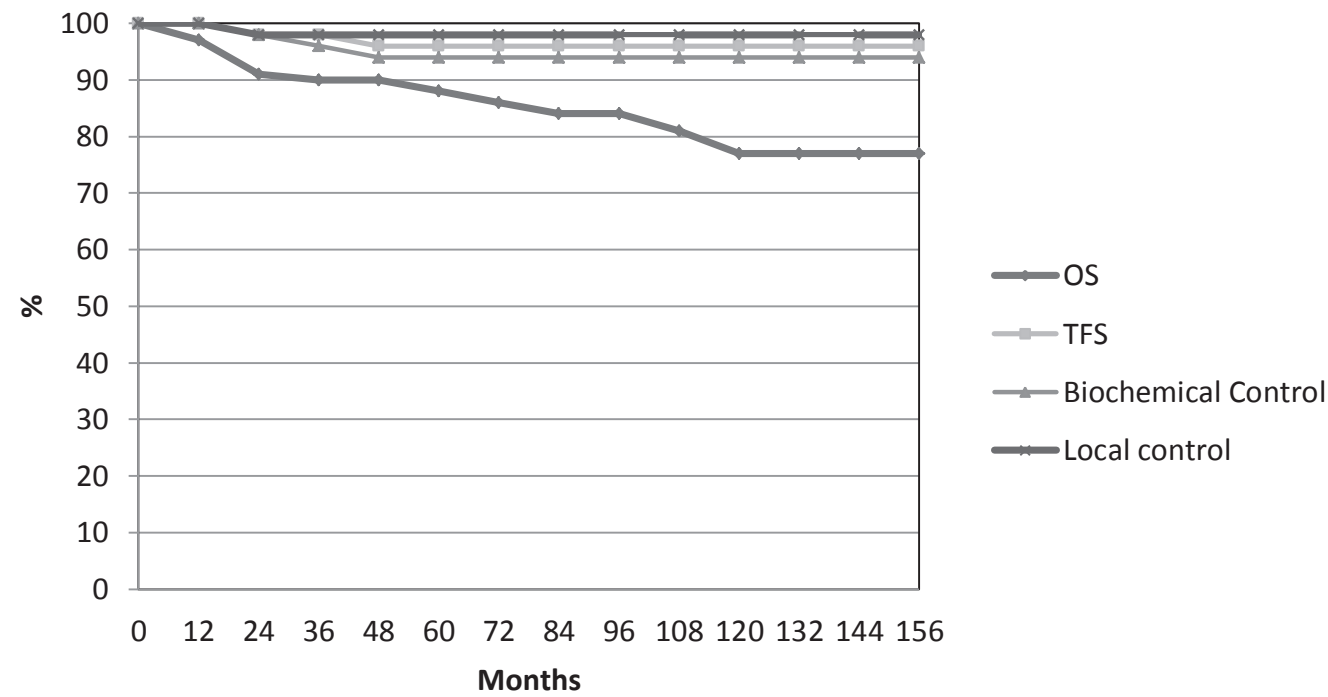

incontinence reported incontinence after brachytherapy (1.7\%). Acute urinary retention was seen in $1(1.7 \%)$ patients, requiring a temporary post-implant bladder catheter. Late urinary retention occurring more than two year after treatment was reported in $1(1.7 \%)$ patients.

The chronic genitourinary complains grade I were 7\% and grade II, 10\%. A patient had late urethral stricture, requiring urethral dilations.

\section{Lower Gastrointestinal Toxicity}

At six months $94 \%$ of patients reported no change in bowel function.

The incidence of rectal ulceration and/or recto-urethral fistula (Toxicity grade III-IV) has been observed in 2 patients (3.5\%) after rectal biopsy.

Intermittent rectal bleeding was reported in 3 patients (5\%). In 6 patients (11\%) transperineal hyaluronic acid injection into the peri-rectal fat was used to consistently displace the rectal wall away from the radiation sources; no mucosal damage and no macroscopic rectal bleeding were observed in this group.

No patients with perineal pain were reported.

\section{Sexual function}

Of the 17 (30\%) patients who were potent preoperatively, $82 \%$ were potent postoperative- ly. Potency was defined as the ability to achieve an erection that was sufficient for intercourse.

\section{DISCUSSION}

Our encouraging results are in concordance with the experience of other institutions (1519). Multivariate and univariate analyses show that the pretreatment PSA level, Gleason score and $T$ stage were not a significant variable for biochemical control. In the present series, hormonal ablative treatment was given for 3-4 months and did not improve biochemical control.

In our series the incontinence grade 1 rate prior to brachytherapy was $9 \%$ (5 patients) and grade II, 3\% (2 patients) but incontinence chronic toxicity TURP-related after brachytherapy was reported only in a patient (1.7\%). Late urethral stricture was reported in $1(1.7 \%)$ patients.

Moran et al. (20) analyzed 171 patients with $\mathrm{T} 1 \mathrm{a}-\mathrm{T} 1 \mathrm{~b}$ prostate cancer who underwent prior TURP. The mean urinary function and bother score for the entire study group was $83.5 \pm 19.5$ and $82.5 \pm 23.7$, respectively. Multivariate analysis revealed higher pretreatment International Prostate Symptom Scores to have significant negative impact on urinary function and bother scores. They concluded that it is feasible LDR brachytherapy in 
selected patients with prior TURP, with low impact on urinary function and bother scores.

Wallner et al. (21) in 19 patients reported a $6 \%$ incontinence rate in a TURP patient group.

Stone et al. (22) suggest that brachytherapy can be safely performed with a low risk of urinary incontinence if a real-time method combined with peripheral loading is used, but they point out that it could result in a higher risk of urinary incontinence.

Ramirez et al. evaluated urinary incontinence in 16 patients with prior TURP and find lower urinary tract symptoms or urinary incontinence after an average of 30 months (23).

Cesaretti et al. (24) evaluated prostate brachytherapy dosimetry outcomes relative to the transurethral resection of the prostate in $\mathbf{7 3}$ patients and they concluded that a visible residual TURP cavity ( $\geq 10 \%$ of a prostate volume) did not appear to be a statistically significant hindrance to proper dosimetric outcome.

Salembier C et al. (25) evaluated prospectively in a multicenter setting the ability of centers to perform pre-implant permanent prostate brachytherapy planning with dosimetric goals and constraints based on the Groupe de Curiethèrapie-European Society for Radiotherapy and Oncology guidelines in patients with prior TURP concluding that it is feasible.

Brachytherapy for patients with a prior TURP and early-stage prostate cancer is effective, with long-term biochemical freedom from recurrence independently of the age of the patients (as younger and older patients benefited equally). The present study showed low toxicity when the dose to any segment of the TURP defects is limited to $\leq 100 \%$ of the prescription dose and the actuarial biochemical control was excellent (95\% for patients with Gleason score of $\leq 6)$. The median hospital stay for our patients was $12 \mathrm{~h}$ (6-8) h after implantation; there are no other alternative treatments with a shorter hospital stay.

The present complications rates were in accordance with the experience of other institutions using permanent implants of 125 I (19-24) for patients with prior TURP.

In conclusion, with the present long-term data, using intra-operative planning with real-ti- me dynamic dose calculation with peripheral loading, LDR brachytherapy provides excellent biochemical control rates for patients with localized prostate cancer and prior TURP, and low urinary and gastrointestinal morbidity.

\section{ABBREVIATIONS}
AJCC = American Joint Committee on Cancer
D90 $=$ The dose that covers 90\% volume of CTV
$\mathrm{GU}=$ Genitourinary
PSA = Serum prostate-specific antigen
PTV = Planning target volume
$\mathrm{CTAE}=$ Common Toxicity Criteria for Adverse
Event.
SPSS $=$ Statistical analysis SPSS
SD = Standard desviations
TFS = Tumour-free survival
TRUS $=$ The trans-rectal ultrasound
TURP $=$ Transurethral prostate resection
LDR $=$ Low dose rate

\section{CONFLICT OF INTEREST}

None declared.

\section{REFERENCES}

1. Gerber GS, Thisted RA, Chodak GW, Schroder FH, Frohmuller $H G$, Scardino PT, et al. Results of radical prostatectomy in men with locally advanced prostate cancer: multi-institutional pooled analysis. Eur Urol. 1997;32:385-90.

2. Prada PJ, Juan G, González-Suárez H, Fernández J, Jimenez I, Amón J, et al. Prostate-specific antigen relapse-free survival and side-effects in 734 patients with up to 10 years of follow-up with localized prostate cancer treated by permanent iodine implants. BJU Int. 2010;106:32-6.

3. Pollack A, Zagars GK, Smith LG, Lee JJ, von Eschenbach $A C$, Antolak JA, et al. Preliminary results of a randomized radiotherapy dose-escalation study comparing 70 Gy with 78 Gy for prostate cancer. J Clin Oncol. 2000;18:3904-11.

4. Blasko JC, Ragde H, Grimm PD. Transperineal ultrasoundguided implantation of the prostate: morbidity and complications. Scand J Urol Nephrol Suppl. 1991;137:113-8.

5. Talcott JA, Clark JA, Stark PC, Mitchell SP. Long-term treatment related complications of brachytherapy for early prostate cancer: a survey of patients previously treated. $J$ Urol. 2001;166:494-9. 
6. Foote J, Yun S, Leach GE. Postprostatectomy incontinence. Pathophysiology, evaluation, and management. Urol Clin North Am. 1991;18:229-41.

7. Greene F, Page D, Fleming I, Fritz A, Balch C, Haller D, et al. AJCC Cancer Staging Manual, 6th edn. Chicago: Springer, 2002.

8. Zelefsky MJ, Leibel SA, Gaudin PB, Kutcher GJ, Fleshner $\mathrm{NE}$, Venkatramen ES, et al. Dose escalation with threedimensional onformal radiation therapy affects the outcome in prostate cancer. Int J Radiat Oncol Biol Phys. 1998;41:491500.

9. Bice WS Jr, Prestidge BR, Prete JJ, Dubois DF. Clinical impact of implementing he recommendations of AAPM Task Group 43 on permanent prostate brachytherapy sing 125I. American Association of Physicists in Medicine. Int J Radiat Oncol iol Phys. 1998;40:1237-41.

10. Prada PJ, Juan G, Fernández J, González-Suárez H, Martínez A, González J, et al. Conformal prostate brachytherapy guided by realtime dynamic dose calculations using permanent 125 iodine implants: technical description and preliminary experience. Arch Esp Urol. 2006;59(9):933-40.

11. Nag S, Ciezki JP, Cormack R, Doggett S, DeWyngaert K, Edmundson GK, et al. Clinical Research Committe, American Brachytherapy Society. Intraoperative planning and evaluation of permanent prostate brachytherapy: report of the American Brachytherapy Society. Int J Radiat Oncol Biol Phys. 2001;51:1422-30.

12. Prada PJ, Gonzalez H, Menéndez C, Llaneza A, Fernández $J$, Santamarta E, et al. Transperineal injection of hyaluronic acid in the anterior perirectal fat to decrease rectal toxicity from radiation delivered with low-dose-rate brachytherapy for prostate cancer patients. Brachytherapy. 2009;8:210-7.

13. Roach M 3rd, Hanks G, Thames H Jr, Schellhammer P, Shipley WU, Sokol GH, et al. Defining biochemical failure following radiotherapy with or without hormonal therapy in men with clinically localized prostate cancer: recommendations of the RTOG-ASTRO Phoenix Consensus Conference. Int J Radiat Oncol Biol Phys. 2006;65:965-74.

14. SPSS Manual. Chicago, SPSS Inc. available at http://www. jou.ufl.edu/archive/researchlab/SPSS-Statistcs-Base-UsersGuide-17.0.pdf

15. Joseph J, Al-Qaisieh B, Ash D, Bottomley D, Carey B. Prostate-specific antigen relapse-free survival in patients with localized prostate cancer treated by brachytherapy. BJU Int. 2004;94:1235-8.

16. Zelefsky MJ, Hollister T, Raben A, Matthews S, Wallner KE. Five-year biochemical outcome and toxicity with transperineal CT-planned permanent $\mathrm{I}-125$ prostate implantation for patients with localized prostate cancer. Int J Radiat Oncol Biol Phys. 2000;47:1261-6.
17. Stone NS, Stock RG. 10-year biochemical and local control following real-time I-25 prostate brachytherapy. J Urol 2003;169 (Suppl.).

18. Ragde H, Elgamal AA, Snow PB, Brandt J, Bartolucci AA, Nadir BS, et al. Ten-year disease free survival after transperineal sonography-guided iodine-125 brachytherapy with or without 45-gray external beam irradiation in the treatment of patients with clinically localized, low to high Gleason grade prostate carcinoma. Cancer. 1998;83:9891001.

19. Grado GL, Larson TR, Balch CS, Grado MM, Collins JM, Kriegshauser JS, et al. Actuarial disease-free survival after prostate câncer brachytherapy using interactive techniques with biplane ultrasound and fluoroscopic guidance. Int J Radiat Oncol Biol Phys. 1998;42:289-98.

20. Moran BJ, Stutz MA, Gurel MH. Prostate brachytherapy can be performed in selected patients after transurethral resection of the prostate. Int $\mathrm{J}$ Radiat Oncol Biol Phys. 2004;59:392-6.

21. Wallner K, Lee $H$, Wasserman S, Dattoli M. Low risk of urinary incontinence following prostate brachytherapy in patients with a prior transurethral prostate resection. Int $J$ Radiat Oncol Biol Phys. 1997;37:565-9.

22. Stone NN, Ratnow ER, Stock RG. Prior transurethral resection does not increase morbidity following real-time ultrasound-guided prostate seed implantation. Tech Urol. 2000;6:123-7.

23. Claros DR, Correia JA, Ferreira LR, Vaz FP. [Retrospective evaluation of urinary continence in patients submitted to brachytherapy after transurethral resection of the prostate gland]. Actas Urol Esp. 2009;33:356-60.

24. Cesaretti JA, Stone NN, Stock RG. Does prior transurethral resection of prostate compromise brachytherapy quality: a dosimetric analysis. Int $\mathrm{J}$ Radiat Oncol Biol Phys. 2004;60:648-53.

25. Salembier C, Rijnders A, Henry A, Niehoff P, André Siebert F, Hoskin P. Prospective multi-center dosimetry study of low-dose lodine-125 prostate brachytherapy performed after transurethral resection. J Contemp Brachytherapy. 2013;5:63-9.

Correspondence address:

Pedro J. Prada, MD

Department of Radiation Oncology Hospital Universitario Marques de Valdecilla

C/ Avd. Valdecilla s/n Santander 39008, Cantabria, Spain Fax:+34 942 202-726 E-mail:pprada@telecable.es 